\title{
Quaderni
}

QUADERNI Communication, technologies, pouvoir

83 | Hiver 2013-2014

Le public de la danse contemporaine

\section{Erik Neveu, Les mots de la communication politique}

\section{Benoit Lafon}

\section{OpenEdition}

Journals

Édition électronique

URL : http://journals.openedition.org/quaderni/778

DOI : 10.4000/quaderni.778

ISSN : 2105-2956

\section{Éditeur}

Les éditions de la Maison des sciences de l'Homme

\section{Édition imprimée}

Date de publication : 5 janvier 2014

Pagination : 119-122

\section{Référence électronique}

Benoit Lafon, «Erik Neveu, Les mots de la communication politique », Quaderni [En ligne], 83 | Hiver 2013-2014, mis en ligne le 01 janvier 2016, consulté le 25 septembre 2020. URL : http:// journals.openedition.org/quaderni/778; DOI : https://doi.org/10.4000/quaderni.778 


\section{Compte rendu}

\section{Les mots de la communication politique}

\section{Erik Neveu}

Presses universitaires du Mirail, Toulouse, 2012

par Benoit Lafon

Université de Grenoble

GRESEC

La collection «Les Mots de», publiée aux Presses Universitaires du Mirail et dirigée par Marlène Coulomb-Gully, se propose, dans son quatrième opus, d'aborder la communication politique avec le concours d'un auteur actif et reconnu de longue date sur la question, Erik Neveu. Le projet est utile et bienvenu, tant la question de la communication politique sous ses formes professionnalisées et technicisées hante l'activité politique depuis plusieurs décennies.

Ainsi, ce petit livre, au format digest, se veut être selon l'éditeur un abécédaire, un inventaire, indispensable « aux étudiants, aux enseignants, à tout public cultivé ou simplement curieux » ( $4^{\mathrm{e}}$ de couverture). Erik Neveu lui-même, dans un avant-propos destiné à clarifier la notion de « communication politique » et ses acceptions diverses selon les publics considérés, détaille les ambitions de ce «petit dictionnaire », à savoir rendre accessibles les savoirs académiques, en même temps qu'intégrer le point de vue des acteurs sociaux impliqués.

En effet, on retrouve bien là certaines spécificités de l'approche de la communication politique défendue par E. Neveu depuis trois décennies. Celle-ci se développe autour de trois idées ( "thématiques-sentiers »), que l'auteur rappelle avant de se livrer à son travail de définition. Première idée, celle de la consubstantialité de la communication aux activités politiques, idée déjà défendue en 1992 par $\mathrm{E}$. Neveu dans un «refus de contribution au dictionnaire des idées reçues $»^{1}$, que le présent ouvrage tend à prolonger. Nombreuses sont les définitions tendant à resituer l'activité politique dans 
sa dimension anthropologique et, de fait, communicationnelle (cf. article « pipolisation » par ex., p. 81). Si les croyances sont répandues sur une nouveauté du phénomène communicationnel en matière politique, elles ne le sont pas moins - c'est la deuxième idée posée par E. Neveu - sur le plan des effets supposés et prêtés aux techniques sans cesse renouvelées de la communication contemporaine. À ce titre, le choix d'un article consacré à la « Magie », s'il peut surprendre de prime abord, n'en est pas moins stimulant par la référence à Mauss. L'on retrouve ici encore un questionnement sur la place contemporaine des activités liées à la communication, véritable secteur social et économique porté par la magie de la croyance, dont les racines avaient été questionnées dans Une société de communication ?. Enfin, la troisième idée, celle des interdépendances entre familles d'acteurs, se fonde sur une vision de la communication politique en tant que phénomène émergeant des interactions sociales. Là encore, l'auteur avait en 1991 avec Rémy Rieffe $l^{2}$ souligné les " effets de réalité des sciences de la communication ", ou comment les savoirs académiques circulent, contribuant à produire des expertises, voire des «munitions argumentatives sur un dossier » (article « Chaînes d'interdépendance », p. 20).

Toutes ces idées et thématiques reflètent la pensée de l'auteur qui est à la base de ce livre. Cependant, ce livre atteint-il son objectif ? Parvient-il à donner la « vue panoramique » $\left(4^{\mathrm{e}}\right.$ de couverture $)$ recherchée par l'auteur ?

Si la réponse semble être positive en raison de l'étendue des questions abordées, le format éditorial de l'ouvrage - un dictionnaire - impose ses limites. Concepts, techniques et vulgate sont ici mêlés sans que l'on saisisse toujours bien le niveau auquel le terme défini renvoie. La figure du dictionnaire est certes pratique, mais en l'état il lui manque un appareil de références pour chaque définition, un mode d'identification pour saisir quel est l'univers du terme défini, qui pourrait alors être relié à une typologie plus nette. Ce format d'abécédaire réduit et destiné à des profanes nécessiterait d'autant plus la présence de métadonnées venant compléter les définitions. Un détour étymologique dans les définitions serait ainsi bienvenu, permettant de mieux répondre aux trois idées précédemment évoquées. Cela étant, par l'étendue des questions traitées et par la rédaction de définitions biens senties et bien amenées, Erik Neveu se tire fort bien de la gageure de produire un dictionnaire à la portée de tous, présentant la communication politique dans une perspective propre aux sciences humaines et sociales.
1. Erik Neveu, « La communication politique : petit refus de contribution au dictionnaire des idées reçues ", Cinémaction $\mathrm{n}^{\circ}$ 63, 1992, pp. 161-167.

2. Erik Neveu, Rémy Rieffel, "Les effets de réalité des sciences de la communication ", Réseaux, Volume 9, Numéro 50, 1991 , pp. $11-40$. 
L'article « Médiacratie » (pp. 70-71) vient ainsi parfaitement illustrer cette tension entre définitions riches à la portée de tous et aplanissement des niveaux de discours. En deux pages parfaitement argumentées s'appuyant sur des enquêtes reconnues, l'auteur déconstruit un mythe et pose une série d'interrogations stimulantes au cœur des débats politico-médiatiques contemporains. Ce faisant, il produit son propre effet de réalité, son propre « cadrage » (cf. définition, p. 17) d'une idée discutée et discutable, contribuant par làmême à sa conceptualisation. 
